\title{
Philosophiques
}

\section{La délibération circonstancielle en théorie démocratique}

\section{Learry Gagné}

Volume 29, numéro 2, automne 2002

La démocratie délibérative

URI : https://id.erudit.org/iderudit/006259ar

DOI : https://doi.org/10.7202/006259ar

Aller au sommaire du numéro

Éditeur(s)

Société de philosophie du Québec

ISSN

0316-2923 (imprimé)

1492-1391 (numérique)

Découvrir la revue

Citer cet article

Gagné, L. (2002). La délibération circonstancielle en théorie démocratique. Philosophiques, 29(2), 327-350. https://doi.org/10.7202/006259ar

\section{Résumé de l'article}

La démocratie délibérative s’oppose fréquemment à une conception rationnelle du citoyen qui, selon ses théoriciens, ne permet pas d'atteindre une authentique démocratie. Pourtant, la démocratie délibérative souffre elle-même de difficultés théoriques importantes dont une partie des solutions a déjà été abordée dans la théorie du choix rationnel. Nous voulons montrer que le choix rationnel, dans sa version "étendue ", peut servir à améliorer la démocratie délibérative sans pour autant en ébranler ses fondements. Nous effectuerons d'abord un survol de la démocratie délibérative, en insistant sur les questions de formation et d'expression des préférences des citoyens dans l'agora. Nous y découvrirons certaines difficultés théoriques que nous tenterons par la suite de résoudre par un ensemble de mécanismes issus de la théorie du choix rationnel. Parallèlement, nous verrons que la délibération raisonnable est possible dans un paradigme rationnel, que nous appelons la " délibération circonstancielle ».
Ce document est protégé par la loi sur le droit d'auteur. L'utilisation des services d’Érudit (y compris la reproduction) est assujettie à sa politique d'utilisation que vous pouvez consulter en ligne.

https://apropos.erudit.org/fr/usagers/politique-dutilisation/ 


\title{
La délibération circonstancielle en théorie démocratique ${ }^{1}$
}

\author{
LEARRY GAGNÉ \\ UQAM \\ gagnel@ awale.qc.ca
}

\begin{abstract}
Résumé. - La démocratie délibérative s'oppose fréquemment à une conception rationnelle du citoyen qui, selon ses théoriciens, ne permet pas d'atteindre une authentique démocratie. Pourtant, la démocratie délibérative souffre elle-même de difficultés théoriques importantes dont une partie des solutions a déjà été abordée dans la théorie du choix rationnel. Nous voulons montrer que le choix rationnel, dans sa version «étendue», peut servir à améliorer la démocratie délibérative sans pour autant en ébranler ses fondements. Nous effectuerons d'abord un survol de la démocratie délibérative, en insistant sur les questions de formation et d'expression des préférences des citoyens dans l'agora. N ous y découvrirons certaines difficultés théoriques que nous tenterons par la suite de résoudre par un ensemble de mécanismes issus de la théorie du choix rationnel. Parallèlement, nous verrons que la délibération raisonnable est possible dans un paradigme rationnel, que nous appelons la « délibération circonstancielle».
\end{abstract}

\begin{abstract}
Deliberative democracy frequently rejects the rational citizen model since, according to its proponents, it would not yield a proper democracy. And yet, deliberative democracy contains important theoretical difficulties, some of which have been dealt with by rational-choice theory. We intend to show that a "broad" version of rational-choice theory can actually improve deliberative democracy without condemning its foundations. First, we will briefly examine deliberative democracy, focusing on the formation and expression of citizens' preferences in the agora. Some theoretical problems will be identified; several mechanisms drawn from rational-choice theory will be used to try to solve them. Simultaneously, we will see that reasonable deliberation is possible in a rational-choice paradigm, in a model I call "circumstantial deliberation".
\end{abstract}

\section{1- Introduction}

La démocratie délibérative est une théorie politique jouissant d'une forte popularité depuis plusieurs années. Elle a pour but d'élaborer les conditions de la pratique de la démocratie tel que les lois et les décisions produites par la délibération publique seront le plus juste possible pour la collectivité. C'est avant tout une théorie idéale, proposant comment les citoyens devraient se comporter afin d'en arriver à la société juste. Bien qu'il existe de nombreuses variantes de la démocratie délibérative, elles supposent toutes un citoyen «raisonnable» : honnête, prédisposé à l'argumentation, ouvert aux opinions des autres et visant le bien-être de la collectivité; elles supposent aussi que ce

1. Une version préliminaire de ce texte a été présentée au Carrefour Étudiant Philosophique, UQ AM , 14-15 février 2002. Je remercie les participants de ce colloque pour leurs commentaires, et tout spécialement Dominique L eydet pour son aide précieuse à la rédaction de la version finale. 


\section{8 - Philosophiques / Automne 2002}

citoyen ait la possibilité de participer à la délibération démocratique d'une manière libre et égale. $M$ ais comme la démocratie délibérative se penche sur un problème social concret - la pratique de la démocratie - , elle se doit de respecter un minimum de réalisme. II est clair qu'un ensemble de propositions normatives inapplicables n'a d'intérêt pour personne. La théorie va donc s'aventurer au-delà de l'éthique pour tenter de modéliser l'interaction sociale rencontrée dans l'agora. N otre hypothèse est que ces modèles rencontrés en démocratie délibérative ne sont pas satisfaisants, au sens où ils règlent un peu trop rapidement des questions pourtant complexes telles que la recherche du consensus, la «force du meilleur argument», et d'autres que nous aborderons tout au long de cet article. Cette théorie, bien qu'elle offre des principes normatifs cohérents et certainement pas dénués d'intérêt, n'aborde pas adéquatement les questions d'application.

Une autre théorie politique en vogue s'occupe précisément de la pratique actuelle de la démocratie: c'est le choix social, une théorie descriptive et explicative fondée sur le choix rationnel qui se penche sur les interactions entre individus rationnels (et souvent égoïstes) cherchant à maximiser leurs intérêts dans l'arène politique. Les démocrates délibératifs ont toujours critiqué cette approche, observant que des individus purement rationnels, maximisant leur utilité dans l'arène politique, n'atteindront jamais la société juste. Pourtant, la rationalité n'est pas incompatible avec la délibération, si l'on adopte une définition large de la rationalité qui accepte, notamment, les comportements altruistes et I'influence des normes sociales. Partant de cette définition, nous allons aborder ce que nous nommerons la délibération circonstancielle, soit un ensemble de mécanismes sociaux ouvrant la voie à la possibilité de délibération parmi des citoyens rationnels. N ous allons donc chercher à voir comment de tels citoyens pourraient en arriver à adopter les comportements prescrits par la démocratie délibérative. $L a$ délibération circonstancielle interpelle les démocrates délibératifs à deux niveaux : d'abord ses mécanismes sociaux leur fournissent des outils théoriques utiles à la modélisation des interactions sociales et de plus, la démonstration que la délibération rationnelle est possible devrait enrichir cette théorie et ouvrir la voie à des variantes plus réalistes. Avant d'aborder les mécanismes de la délibération circonstancielle, nous allons nous pencher sur les difficultés rencontrées par la démocratie délibérative dans l'application de ses principes normatifs.

\section{2- La pratique délibérative}

Dans cette section nous nous intéresserons aux comportements des citoyens à l'intérieur des modèles politiques préconisés par diverses variantes de la démocratie délibérative. Cet examen de la pratique délibérative nous permettra d'évaluer comment ces théories effectuent le passage crucial de la prescription normative vers l'application concrète. II ne s'agit pas d'une étude empirique de la délibération, mais bien d'une modélisation abstraite de l'agora fondée sur certaines conceptions du citoyen individuel et des procédures 
démocratiques. $\mathrm{N}$ ous verrons comment certains principes fondamentaux de la démocratie délibérative tels l'argumentation raisonnable, l'égalité, la réciprocité, et d'autres prennent vie dans ces modèles. L'étude de l'application de ces principes normatifs peut prendre de multiples formes. Étant donné que nous avons choisi d'aborder ces modèles délibératifs d'un point de vue rationnel, nous nous concentrerons sur deux aspects chers à la théorie du choix rationnel, soit la formation et l'expression des préférences individuelles. Cette discussion tournera autour du concept d'autonomie des préférences, un concept relié à la liberté individuelle préconisée par la démocratie délibérative. N ous débuterons par la question de la formation autonome des préférences: le consensus s'atteint-il par réflexion libre et rationnelle ou par conformisme à l'opinion majoritaire? $N$ ous passerons ensuite à la question de leur expression autonome : jusqu'à quel point l'exigence d'égal ité contraint-elle les arguments que le citoyen peut avancer? N ous verrons que sur ces questions, certaines variantes importantes de la démocratie délibérative exhibent des contradictions théoriques ainsi que des effets pervers de nature psycho-sociologique.

\subsection{La formation des préférences}

Dans la théorie du choix rationnel, l'action est déterminée par les désirs et les croyances de l'agent. Selon le modèle de J on Elster que nous allons utiliser tout au long de cet article, pour qu'une action soit substantiellement rationnelle les désirs doivent être autonomes et la formation des croyances doit exhiber un certain jugement. De plus, les liens entre désirs et croyances sont sujets à évaluation. Commençons par l'autonomie des désirs. Les phénomènes les plus courants d'irrationalité dans la sphère politique concernent les comportements conformistes (ou anticonformistes), le respect irréfléchi des traditions ou la révolte contre celles-ci, etc. Aux deux extrêmes, les préférences «adaptatives», soit la correspondance parfaite avec la situation actuelle, perpétuent le statu quo social alors que les préférences « contre-adaptatives», illustrées par le dicton de l'herbe toujours plus verte sur le terrain du voisin, aboutissent à un choix social impossible à implémenter ${ }^{2}$ ou du moins très instable ${ }^{3}$. Ces phénomènes sont constitutifs d'une réalité indéniable concernant la formation de n'importe quelle préférence, soit l'influence de la société par l'éducation et I'habitude. Aucun être humain ne peut se prétendre complètement immunisé des valeurs de la société dans laquelle il vit. Les individus auraient

2. Elster, 1986, p. 109-110.

3. Dans la logique des ensembles, avec l'ensemble des préférences $P$ d'un individu et I'ensemble faisable $F$ nous avons l'adaptation lorsque $P \cap F=P$ et la contre-adaptation lorsque $P \cap F=\varnothing$. Dans sa définition de l'autonomie, Elster demande que $P$ et $F$ s'entrecoupent mais pas entièrement. L'instabilité de l'implémentation sociale des préférences contre-adaptatives s'illustre de la façon suivante : lorsque le gouvernement implémente des préférences formées de telle manière, il se trouve à modifier $\mathrm{F}$ afin d'y inclure $\mathrm{P}$ mais alors les citoyens contre-adaptatifs (irrationnels) vont rejeter la nouvelle donne en exprimant de nouvelles exigences au-delà de $F$. 
donc un penchant naturel pour la perpétuation des valeurs et des institutions existantes. Par exemple, I'adoption du vote proportionnel est beaucoup plus difficile à obtenir dans un pays avec une longue tradition de démocratie directe par circonscriptions que dans un pays sans véritable tradition démocratique. Bien que ne constituant pas une irrationalité, cet effet de préservation d'acquis ${ }^{4}$ prouve que les individus ne forment pas leurs préférences en toute liberté.

Débutons par la formation des préférences en démocratie délibérative. Par censure de groupe $e^{5}$ on entend les divers modes de pression sociale contre les prises de positions ouvertement égoïstes. La délibération se fie notamment sur la « force civilisatrice de l'hypocrisie» pour convertir les individus égoïstes. Postulant l'existence d'une norme sociale contre l'expression de préférences égoïstes en public, ce mécanisme stipule qu'un individu égoïste, à force de s'exprimer d'une façon faussement impartiale, en viendra à sincèrement adopter ces principes ${ }^{6}$. Bien que justifiables en termes de justice sociale, de telles préférences ne répondent pas adéquatement au critère rationnel d'autonomie. Si le mécanisme opérationnel est la réduction de dissonances, il devient difficile de croire que de telles préférences ont été intentionnellement formées ${ }^{7}$. Difficile donc de faire la part des choses entre préférences intentionnelles ou non, entre préférences « réelles» ou stratégiques. Aussi, la distinction entre intérêts privés et publics semble trop simpliste. Par exemple, lorsqu'un groupe exclu de l'agora exige sa place dans l'espace public, il le fait au nom de ses intérêts qui, on le suppose, ne sont pas représentés adéquatement ${ }^{8}$.

La persuasion, tout comme la censure, se bute aussi à l'autonomie des préférences. Les théoriciens délibératifs insistent sur une persuasion « rationnelle» fondée sur un débat raisonnable et un ajustement volontaire et justifié par l'individu de ses positions initiales. Toutefois, il n'existe aucun moyen sûr de distinguer entre une telle forme de persuasion et un simple mimétisme ou un effet d'entraînement de la masse ${ }^{9}$, les deux dernières formes étant manifestement irrationnelles. La persuasion peut aussi induire une nouvelle inégalité substantielle car certains individus sont plus versés que d'autres dans l'art oratoire et disposent donc d'un avantage sur les autres. Bien que certains aient tenté de critiquer cette inégalité ${ }^{10}$, il demeure extrêmement difficile de voir comment la force persuasive pourrait être uniformisée préalablement à la discussion. La démocratie délibérative ne peut espérer une transformation massive des préférences par la simple discussion publique ${ }^{11}$.

4. Endowment effect, Sunstein, 1993, p. 199.

5. L'emploi du terme «censure» tout au long de ce texte n'implique pas de connotations négatives, ni un jugement de valeur envers la démocratie délibérative. Toute théorie politique encadre d'une certaine manière les faits et gestes des citoyens, c'est ce que le terme cherche à saisir.

6. Elster, 1994, p. 190.

7. Elster, 1986, p. 113 ; Johnson, 1998, p. 172.

8. Johnson, 1998, p. 174 ; Knight, Johnson, 1994, p.288.

9. Elster, 1986. P. 116-17.

10. Young, 1996.

11. Johnson, 1998, p. 174. 
L'uniformisation des préférences n'étant pas moralement désirable si elle implique la violation de l'autonomie individuell ${ }^{12}$, nous sommes alors confrontés à une pluralité des conceptions du social, ce que R awls appelle les doctrines compréhensives. Le conflit doctrinaire prend une toute autre dimension que la simple divergence d'intérêts; il met en présence des valeurs fondamentales, telles les croyances religieuses, qui ne peuvent être objets de négociations. Tout ce que l'on peut espérer pour éviter le conflit, c'est la tolérance. Chez Rawls, le citoyen idéal est qualifié de «raisonnable», un concept assez près de celui rencontré habituellement en démocratie délibérative, incluant en particulier la tolérance et l'impartialité. Les val eurs sociales de tels citoyens se nomment, cela va de soi, doctrines compréhensives raisonnables. Le conflit doctrinaire déraisonnable ne devrait donc pas survenir dans un système délibératif bien ordonné car, bien sûr, les doctrines déraisonnables y sont éliminées dès le départ ${ }^{13}$.

$M$ alheureusement, la réalité $n$ 'est pas si facile et les conflits impliquant des doctrines déraisonnables y seront bien souvent insolubles. D'abord, ce qui est raisonnable chez Rawls constitue en soi une doctrine particulièr $e^{14}$. M ême si Rawls prétend que ses principes de justice servent de fondation aux doctrines raisonnables ${ }^{15}$, cela ne fait que repousser le problème à un niveau supérieur. Ancrer ses préférences exprimées à une doctrine comporte un avantage de crédibilité, car on abandonne avec beaucoup plus de peine une valeur morale qu'une valeur d'utilité. Cela peut aussi servir la communauté en forçant un débat de fond sur les problèmes sociaux plutôt qu'une lutte d'intérêts superficiels. $M$ ais son principal effet pervers sera de durcir les positions jusqu'à l'impasse politique, surtout si l'engagement préalable se prend en public : «(...) les mêmes motifs qui font que les interlocuteurs ont adopté d'emblée une attitude d'argumentation plutôt qu'une attitude de négociation les incitera également à tenir leurs engagements» et à réduire les chances de succès de la délibération ${ }^{16}$. Comme nous le remarquons fréquemment dans nos assemblées politiques, la publicité des débats amène les différents groupes à vouloir se démarquer idéologiquement; ils tendent ainsi vers la rhétorique ${ }^{17}$.

Gutmann et Thompson répondent à ce problème en suggérant le principe délibératif de réciprocité. Face à un désaccord moral fondamental, les citoyens mus par l'idéal de réciprocité abandonnent leurs intérêts personnels et, autant que possible, leurs arguments moraux incompatibles avec la position morale opposée. Sans nécessairement résoudre le conflit, ils atteignent à tout le moins un certain niveau de respect mutuel ${ }^{18}$. Ces théoriciens délibératifs admettent que

12. O u même un doute lancinant sur cette autonomie, car dans bien des cas il nous sera impossible de faire la part des choses.

13. Rawls, 1995, ch. II.

14. Johnson, 1998, p. 168-69.

15. Rawls, 1995, p. 183.

16. Elster, 1994, p. 248.

17. Elster, 1986, p. 118.

18. Gutmann, Thompson, 1996, ch. 2. 


\section{2 - Philosophiques / Automne 2002}

les conflits peuvent se durcir et même augmenter en nombre sous ces conditions, mais tout devrait relativement bien se passer en postulant des citoyens de bonne foi. La lacune fondamentale d'une telle proposition, c'est qu'en politique la bonne foi des participants résout énormément de conflits ${ }^{19}$. Évidemment ce n'est pas là un postulat suffisant, même s'il est longuement décortiqué avec le principe d'«accommodement moral » 20,21 . N ous retrouvons aussi cette bonne foi dans ce court passage: « $M$ oral argument can arouse moral fanatics, but it also combats their claims on their own terms ${ }^{22}$. On voit mal comment on pourrait «combattre» les fanatiques au niveau argumentatif sans supposer qu'ils soient eux-mêmes ouverts au débat, ce qui est une contradiction. Cette théorie doit spécifier une autre méthode plus convaincante de négociation avec les fanatiques ou même tout citoyen qui ne discute pas pleinement de façon réciproque. Bien que la volonté des démocrates délibératifs sur la question des conflits politiques soit de favoriser la tolérance et le respect mutuel - objectifs parfaitement louables en soi - , force nous est de constater que ce type de délibération peut augmenter et rendre plus insolubles les conflits; en cela il n'est pas certain que la solution délibérative soit la meilleure.

\section{2 - L'expression des préférences}

A utant la théorie du choix social que la théorie délibérative élaborent minimalement des procédures justes d'élaboration de politiques gouvernant la société. Toutes deux se fondent sur un individualisme méthodologique. Du côté épistémologique, elles dotent l'individu de certaines motivations intentionnelles encadrées par des critères fondamentaux de justice sociale. Les politiques adoptées trouvent leur justification dans la procédure les ayant engendré. La raison d'être des théories est la même : les citoyens ont le pouvoir de forger de façon libre et intentionnelle une société viable, au double sens de capacité de survie de la société et de bonne vie pour ses membres. Les deux théories veulent absolument se distinguer des conceptions compréhensives du politique où le «philosophe-roi » détermine lui-même les institutions sociales en faisant fi des préférences individuelles de ses sujets, car évidemment cette méthode est foncièrement antidémocratique. Toutefois, ces théories vont quand même instaurer des limites aux arguments pouvant être présentés en public. Pour ce qui est du choix social, ces limites se veulent minimales; des

19. Cohen semble partager cette foi : «The structure of discussion, aimed at solving problems rather than pressuring the state for solutions, would encourage people to find terms to which others can agree. And that would plausibly drive argument and proposed actions in directions that respect and advance more general interests» (Cohen, 1996, p. 113).

20. Gutmann, Thompson, 1996, p. 79-85.

21. N ous retrouvons dans ce concept des prescriptions de comportement comme la cohérence de ses principes moraux, la reconnaissance de l'autre, l'ouverture d'esprit ou la minimisation des points de désaccord. Bien entendu, adopter une attitude morale de résolution de conflits va réduire les conflits et nous aider à vivre avec le résiduel insoluble potentiel, mais audelà de cette vérité plate, Gutmann et Thompson ne nous en disent pas beaucoup plus.

22. Gutmann, Thompson, 1995, p. 106 
arguments violant l'intégrité et la dignité de la personne, comme des propos racistes, violents, etc., seront supprimés mais bien d'autres arguments, même fondés sur la position de pouvoir du locuteur, seront admis. II n'en est pas de même en démocratie délibérative, qui se veut beaucoup plus contraignante.

A morçons cette discussion avec la définition de R aw Is de la justice procédurale. II nous offre quatre types. D'abord, la justice procédurale parfaite propose un critère d'évaluation des fins indépendant de la procédure, ainsi qu'une procédure telle que des individus rationnels atteindront de plein gré le résultat escompté. II va sans dire que cette coïncidence entre justice et intérêt particulier est plutôt inusitée; Rawls propose donc la justice procédurale imparfaite où la procédure ne peut garantir la convergence vers le résultat demandé par le critère de justice substantiel. Dans les deux cas, le résultat correct est déterminé à l'avance, la question devient de savoir comment l'atteindre démocratiquement. En justice procédurale pure, le résultat est indéterminé et trouvera sa justification dans la justesse de la procédure suivie. Rawls spécifie que ce type de justice doit être complété par des institutions justes, sinon on risque le chaos; sel on lui, celles-ci sont choisies dans la position originelle, alors que le choix social impose des institutions libérales ${ }^{23}$. Une justification supplémentaire apparaît en justice procédurale quasi-pure, soit le spectateur impartial respectant certains critères substantiels de justice. Le résultat de la procédure est ainsi comparé à ce que des citoyens hypothétiques obéissant à une justice substantielle auraient choisi. Ce résultat hypothétique ne se veut pas unique, mais couvre un certain éventail de résultats justes ${ }^{24}$. C'est une façon originale de réintroduire le substantiel dans un principe qui avait pour but d'en limiter la portée.

Plusieurs démocrates délibératifs vont employer cette stratégie de la double légitimation consistant à imposer un critère supplémentaire de légitimation du résultat, au-delà du respect de la procédure juste. Pour Gutmann et Thompson, la délibération doit se dérouler en contexte de respect mutuel et de réciprocité, soit l'attention portée aux arguments d'autrui, la disposition à réviser ses propres arguments, etc. C'est une forme de justice substantielle présente dans la procédure même. Le résultat n'est légitime que si les citoyens ont respecté les critères de réciprocité ${ }^{25}$. La procédure juste ne suffit pas : «From a deliberative perspective, the problem with relying on bargaining as a substitute for moral reasoning, even within political institutions that are fully just, is that it rests on too thin a conception of what citizens owe one

23. Pour Gutmann et Thompson (1995, p. 99), on retrouve autant dans les fondements substantiels de R awls («justice constitutionnelle») que dans ceux du choix social («justice procédurale») des principes nécessaires à la procédure juste (droit de parole, etc.) et des principes extra-procéduraux nécessaires à la survie de la société (revenu minimum, etc.). Ces principes servent de contraintes à la procédure. Les deux diffèrent lorsque Rawls propose en plus des principes non-nécessaires à la société et non-contraignants.

24. Rawls, 1971, p. 85-87, p. 362,

25. Gutmann, Thompson 1995, p. 104-6. 


\section{4 - Philosophiques / Automne 2002}

another in an increasingly interdependent society ${ }^{26}$. Cohen propose une version légèrement différente : si deux procédures donnent exactement le même résultat, on doit préférer celle qui laisse le plus de place à la délibération, «because of the greater confidence in the deliberative character of the process and the increased confidence in the outcomes that results ${ }^{27}$. O n remarquera qu'il est ici question de confiance et non de légitimité, mais c'est passablement la même chose.

Gutmann et Thompson adoptent également une forme de justice procédurale quasi-pure. Ils nous offrent un exemple de délibération se déroulant dans un train : les passagers doivent décider s'ils ont le droit de fumer à l'intérieur du wagon. Quelques uns (une minorité) invoquent l'argument de la détérioration de la santé de tous, malgré cela le vote est pris et la permission de fumer l'emporte. Les auteurs voient là une situation où la procédure majoritaire perd de sa légitimitéz ${ }^{28}$. Cette procédure constitue pour eux une théorie politique dite de «premier ordre», c'est-à-dire qu'elle s'impose en rejetant les théories alternatives. N ous faisons donc face à un désaccord entre deux visions de la politique : la procédure majoritaire et la défense du bienêtre collectif proposée par la minorité. A ucune des deux ne doit l'emporter sur I'autre a priori. La démocratie délibérative est, quant à elle, une théorie de «second ordre» se situant au-dessus des autres théories, dans une position permettant de les juger ${ }^{29}$. En fait, cela ressemble plus à I'argument du spectateur impartial de Rawls jugeant des résultats selon certains principes. $M$ ais ils s'aventurent plus loin. Selon eux, la démocratie délibérative elle-même n'échappe pas à son auto-critique; elle fait donc l'objet de ses propres principes. Bien que ce critère préserve la cohérence de la théorie, il la vide également de son contenu. Tous les principes de la démocratie délibérative se veulent provisoires, et il ne nous reste en définitive que le principe fondamental de réciprocité (qui peut être lui-même rejeté, mais alors ce sera la fin de la démocratie délibérative). C ette technique permet à Gutmann et Thompson d'introduire des principes de justice substantielle sans vraiment avoir à les inclure formellement dans la théorie ${ }^{30}$.

Une troisième variante de la double légitimation est la « validation récursive» de Seyla Benhabib. Partant d'une définition délibérative classique de la procédure juste - délibération libre et raisonnée, égalité morale et politique des citoyens - , elle n'impose aucune restriction aux arguments exprimables

26. Gutmann, Thompson, 1996, p. 58, italiques rajoutées.

27. Cohen, 1986, p. 37.

28. Gutmann, Thompson. 1995, p. 94-95.

29. Gutmann, Thompson, 2000.

30. Le problème épistémologique qui se pose, c'est que les principes de toute théorie, dans tous les domaines de la science, sont essentiellement provisoires. M ême la théorie politique la plus totalitaire et moralement hermétique finit par transformer ses principes au fil du temps. D onc, cette définition particulière de la démocratie délibérative n'apporte rien de concret et sombre dans un profond relativisme. 
dans l'agora et aux résultats conséquents : «Procedures can neither dictate outcomes nor define the quality of the reasons advanced in argumentation nor control the quality of the reasoning and rules of logic and inference used by participants ${ }^{31}$. Ceci représente le premier niveau de délibération. La seconde légitimation fait son apparition au «méta-niveau», une délibération permettant de juger des résultats du niveau précédent. Cette validation récursive permet aux adversaires de la solution démocratiquement proposée de s'y opposer et ainsi de forcer l'élaboration d'une autre solution : « (...) only the freely given assent of all concerned can count as a condition of having reached agreement in the discourse situation ${ }^{32}$.

La double légitimation s'adresse à une difficulté bien connue en démocratie délibérative, la tension entre le citoyen « idéal » raisonnable et le citoyen «réel » dont les actes peuvent dévier de l'idéal de justice. Par souci de réalisme, les partisans d'une forme ou d'une autre de la double légitimation ne veulent pas imposer l'idéalisme au niveau du citoyen mais en fait, ils ne font que repousser cet idéalisme à un niveau supérieur, ce qui n'est guère mieux. Le besoin de deux mécanismes de légitimité dans ces théories doit également être compris à l'intérieur d'une tension entre l'intuition démocratique de laisser les citoyens décider par eux-mêmes dans le cadre d'une procédure juste et I'intuition paternaliste de juger le résultat sel on des critères substantiels extraprocéduraux. Bien qu'ancrée d'une certaine manière dans la procédure, la justice quasi-pure, la réciprocité et la validation récursive émanent d'une justice substantielle, sinon pourquoi ne pas choisir le tirage au sort comme processus décisionnel ? Si le tirage s'effectue dans des conditions équitables, voilà une procédure tout à fait juste. Le fait de préférer la délibération au tirage démontre que l'on recherche l'imposition d'un certain type de procédure, pas seulement une procédure quel conque respectant des critères d'impartialité et d'équité ${ }^{33}$. On attribue souvent à une telle procédure une valeur civilisatrice propre. Les individus «raisonnables», en écoutant les autres et en évaluant leurs points de vues sans préjugés, en viennent à devenir de meilleurs citoyens. La procédure prend la forme d'une fin, pas seulement d'un moyen. Cette fin est nécessairement substantielle.

L'égalité des citoyens constitue une part importante de la justice substantielle délibérative. Cela peut prendre plusieurs formes. II y a d'abord l'égalité politique, le fondement de toute théorie démocratique, soit le droit de vote égal (une personne, un vote), le droit égal de se présenter aux élections, etc. Vient ensuite l'égalité morale, c'est-à-dire l'absence de toute autorité morale formelle dans le débat. Les participants n'ont pas à se plier aux arguments d'autrui seulement à cause de sa supériorité comme membre du clergé, philosophe, etc. Benhabib, par exemple, fonde la démocratie délibérative sur

31. Benhabib, 1996, p.72.

32. Benhabib 1996, p. 79, italiques dans l'original.

33. Cooke, 2000, p.950-52. 


\section{6 - Philosophiques / Automne 2002}

ce qu'elle nomme le «modèle discursif de l'éthique», comprenant l'égalité de participation et de propositions d'arguments et le droit égal de remettre en question les sujets à l'ordre du jour ainsi que les règles délibératives ${ }^{34}$. Rawls maintient à peu près le même propos avec son principe de participation égal $\mathrm{e}^{35}$. II n'est pas question dans ce principe de redistributions économiques ou sociales ${ }^{36}$.

D'autres théoriciens, par contre, vont exiger une égalité plus substantielle. La justification générale est simple: afin d'atteindre une authentique égalité politique, il faut éliminer les facteurs externes conférant un avantage injuste à certains participants, comme la position sociale ou la propriété de ressources économiques. N ous retrouvons de tels principes chez Cohen : «The participants are substantively equal in that the existing distribution of power and resources does not shape their chances to contribute to deliberation, nor does that distribution play an authoritative role in their deliberation ${ }^{37}$. Ses propos semblent signaler une forme d'auto-censure; on laisse ses avantages au vestiaire en entrant dans I'agora. Lorsque les démocrates délibératifs posent l'égalité substantielle en termes d'un idéal de citoyenneté, en érigeant des barrières théoriques à l'expression des préférences, ils ne nous informent aucunement sur les mécanismes permettant d'y arriver. Rawls a bien compris ce problème : «(...) the principle of participation applies to institutions. It does not define an ideal of citizenship; nor does it lay down a duty requiring all to take an active part in political affairs» ${ }^{38}$. Pour lui, I'égalité individuelle substantielle est une question de sociologie politique, pas d'une théorie de la justice ${ }^{39}$. Lorsque Cohen exige une certaine redistribution égalitaire sous forme de financement public des partis, il penche du côté de R awls mais sa volonté de situer l'égalité au niveau du citoyen revient constamment ${ }^{40}$.

La variante la plus répandue de l'égalité substantielle se retrouve dans le principe délibératif habermasien de la «force du meilleur argument». Les arguments devraient être évalués selon leur vérité, leur sincérité et leur valeur morale (souvent l'impartialité), et non selon l'avantage social ou matériel de celui ou celle qui les propose. Ce principe agit comme une forme de censure à la discussion, régulant ce qui peut ou ne peut être avancé comme arguments. Une

34. Benhabib, 1996, p. 68.

35. Rawls, 1971, p. 221-34.

36. II est à noter que chez R awls le principe de participation égale se limite à des questions politiques essentielles comme la formation de la Constitution. Les exigences égalitaires sont beaucoup plus fortes dans la position originelle, mais ceci se veut un exercice abstrait de découverte des principes de justice et non une définition des individus réels dans des discussions concrètes. Rawls demande seulement que les citoyens exhibent en général une disposition morale compatible avec la position originelle, peu importe s'ils I'appliquent ou non (Rawls, 1971, p. 505). Bien sûr, l'application conduira à une société plus juste, mais ce n'est pas une exigence formelle.

37. Cohen, 1989, p. 23.

38. Rawls, 1971, p. 227.

39. Rawls, 1971, p. 226-27.

40. Cohen, 1989, p. 30-32; Cohen, 1996, p. 108-10. 
proposition peut être rejetée si elle n'est pas fondée sur des « raisons acceptables ${ }^{41}$. La démocratie délibérative ne s'intéresse pas aux gens n'appliquant pas le principe de réciprocitét2. L'État peut même intervenir afin de censurer leurs opinions ${ }^{43}$. Cohen allait encore plus loin dans sa théorie du populisme épistémique qu'il adoptait il y a plusieurs années (il a depuis changé d'opinion). Les citoyens ne doivent pas exprimer leurs préférences, mais se limiter plutôt à évaluer les propositions à l'étude selon les critères d'une « volonté générale» correspondant plus ou moins aux principes raw Isiens de justice. II est permis de modifier la procédure si jamais la tentation de recourir aux préférences s'avère trop forte ${ }^{44}$. II faut toutefois admettre que la démocratie délibérative n'endosse pas généralement ce genre de proposition.

O utre le questionnement sur les conséquences d'un tel contrôle sur la démocratie, il existe une autre critique plus axée sur la théorie. Elle est amenée un peu indirectement par Young dans son exposé post-moderniste sur la validité de la «force du meilleur argument ${ }^{45}$. Elle voit dans l'égalisation des participants un danger d'uniformisation, voire de conservatisme. Ainsi, la discussion raisonnable exclurait les voix plus émotives ou encore provenant de cultures ne pratiquant pas le débat «raisonnable» au sens des démocrates délibératifs ${ }^{46}$. Elle s'en prend dans son texte au caractère mâle et Blanc des institutions délibératives proposées par les théories, mais nous pouvons en déduire un autre problème, celui de la relativité des prémisses d'égalité délibérative. En effet, pourquoi s'arrêter à l'égalité des positions sociales ou des ressources? Certains possèdent plus de talent persuasif que d'autres, certains sont davantage attirés vers la compétition intellectuelle, etc. Pour Young, la diversité est une ressource nous permettant de découvrir les besoins réels des autres, pas une variable à niveler ${ }^{47}$. Elle nous amène à réfléchir sur la pertinence de l'argumentation d'égal à égal en démocratie délibérative ${ }^{48}$. Elle soulève également un coin du voile recouvrant la complexité d'une conception adéquate de l'égalité.

$\mathrm{N}$ ous avons vu dans cette section que la sphère politique affecte la formation autonome des préférences, par les effets de préservation d'acquis,

41. Cohen, 1989, p. 22.

42. Gutmann, Thompson, 1996, p. 55.

43. Rawls, 1995, p. 91.

44. Cohen, 1986, p. 34-37.

45. Young, 1996.

46. Young, 1996, p. 122-24. Johnson (1998, p. 166) abonde dans le même sens lorsqu'il observe qu'à prime abord, la désobéissance civile, par son caractère agressif et impertinent, n'a pas sa place en démocratie délibérative même si ses demandes sont parfaitement légitimes.

47. Young, 1996, p. 126-28.

48. Young ne rejette pas vraiment la démocratie délibérative, elle la remplace par la « démocratie communicative» où des individus affirmant leurs différences en viennent à transformer leurs préférences par un processus d'apprentissage et de respect de l'autre. Benhabib (1996, p. 82) se demande avec justesse quelle est la différence entre ce mécanisme et le consensus raisonnable que l'on rencontre habituellement en démocratie délibérative. 


\section{8 - Philosophiques / Automne 2002}

d'entraînement de la masse et de l'adaptation au possible. Cohen a relevé ce problème potentiel en politique, mais selon lui les principes délibératifs, notamment le «pouvoir de la raison», assurent l'autonomie des préférences ${ }^{49}$. II n'y aurait donc pas de problèmes, malheureusement il ne précise pas vraiment comment on en arrive au juste à cette autonomie. L'introduction de considérations rationnelles nous apprend qu'il est impossible de distinguer clairement, lors d'un débat public, entre une préférence authentiquement autonome et un simple effet de conformisme. Le «pouvoir de la raison» ne garantit donc rien. Plus encore, la délibération à grande échelle a tendance à exacerber les mécanismes contrant la formation autonome des préférences ${ }^{50}$, c'est le phénomène bien connu des effets de foule. Des représentants élus ou des leaders émergeants opportunistes peuvent se servir des effets de foule pour assouvir leurs propres fins, par la démagogie et la rhétorique. A fin de se prémunir de ces tendances, il est parfois préférable pour les représentants de se réunir à huis clos. Cette violation du principe de publicité peut être justifiée de façon délibérative lorsque les citoyens décident librement (par vote ou consensus) qu'il serait mieux au nom de la collectivité que les représentants ne soient pas tentés de sombrer dans la démagogi ${ }^{51}$. En effet, la délibération publique et transparente sur des sujets controversés ne se fait généralement pas à tête reposée.

Les concepts de réciprocité et de doctrine compréhensive raisonnable ont comme but de s'accorder avec le «fait du pluralisme», soit la reconnaissance que nous n'arriverons jamais à des choix unanimes de société et que de toute façon il serait moralement indésirable d'en arriver là. Toutefois, ces mêmes principes peuvent conduire à un durcissement idéologique, réduisant ainsi considérablement la portée de la délibération démocratique. Gutmann et Thompson ont reconnu ce problème, mais comme nous l'avons vu, ils ne nous offrent pas de véritables méthodes de résolution. Les différentes variantes de «double légitimité» proposent des mécanismes d'agrégation des préférences individuelles assurant le respect d'une certaine justice substantielle, mais si la démocratie délibérative veut s'engager sur ce terrain extrêmement fertile, il faudrait qu'elle nous offre des mécanismes beaucoup plus élaborés que la « validité récursive» ou son statut de théorie de second ordre. Enfin, nous avons constaté que l'égalité des citoyens dans l'agora est loin d'être un concept simple, car il existe une multitude d'implémentation possible des mêmes critères généraux d'égalité.

\section{3 - La délibération circonstancielle}

Ayant entrevu les conséquences de l'introduction de certains principes rationnels dans la modélisation de l'agora, nous allons maintenant nous pencher plus sérieusement sur les manières dont la théorie du choix rationnel pourrait nous

49. Cohen, 1989 , p. 25-26.

50. Elster, 1998, p. 107-9.

51. Luban, 1996, p. 189-92; Gutmann, Thompson, 2000, p. 176-77. 
venir en aide concernant les questions d'implémentation des principes normatifs de la démocratie délibérative. $D$ 'abord, une précision s'impose. La théorie du choix rationnel n'exige aucunement que l'agent se comporte de façon égoïste. On rencontre souvent cette hypothèse car ce sont les économistes qui se servent le plus du choix rationnel et pour eux, l'agent égoïste génère de meilleurs modèles. $M$ ais dans un domaine comme la politique, on peut appliquer le choix rationnel sans présupposer le caractère égoïste ou altruiste du citoyen. II est donc possible de considérer en choix social, par exemple, des motivations individuelles inspirées des idéaux délibératifs. En fait, on pourrait affirmer grossièrement qu'un système politique fondé sur le choix social et ne comprenant que des participants motivés par l'éthique délibérative serait identique à ce que la démocratie délibérative elle-même propose. Bien sûr, la leçon fondamentale que nous enseigne le choix social est que nous ne pouvons compter sur une telle communion morale. La possibilité de délibération parmi des citoyens rationnels nous permettra de résoudre certaines des difficultés rencontrées plus haut en démocratie délibérative, mais non sans en faire apparaître d'autres. N ous allons d'abord nous intéresser aux motivations individuelles de type délibératif pour ensuite traiter des possibilités d'action stratégique entourant les choix collectifs, le tout dans une perspective rationaliste.

\section{1- Les motivations individuelles}

Les démocrates délibératifs ont fréquemment tendance à opposer le «raisonnable» au «rationnel», menant ainsi au conflit quasi-perpétuel entre démocratie délibérative et choix social ${ }^{52}$. Rawls considère notamment la possibilité d'individus rationnels altruistes, mais en tant qu'individus exprimant des intérêts personnels en tenant compte du bien-être d'autrui. II poursuit : «Ce qui manque aux agents rationnels, c'est la forme particulière de sensibilité morale qui sous-tend le désir de s'engager dans une coopération équitable comme telle $(. ..) »^{53}$. N ous allons tenter de démontrer que cette «sensibilité morale» peut faire partie des motivations de l'agent rationnel et qu'elle peut être provoquée ou facilitée par certains mécanismes socio-psychologiques. Pour ce faire nous allons surtout nous baser sur le modèle d'Elster, qui nous invite à considérer les normes sociales et les émotions comme sources supplémentaires de motivations chez l'agent rationnel.

II existe chez Elster trois catégories de motivations, soit l'intérêt, la raison et la passion ${ }^{54}$. Sa définition de la raison se rapproche beaucoup de celle

52. Le choix social n'est certainement pas innocent dans ce conflit, ainsi le plaidoyer de Sen (1986) pour que ses théoriciens respectent les choix éthiques.

53. Rawls, 1995, p. 79. Gutmann et Thompson formulent la même critique de la rationalité : «Even if citizens were to bargain under conditions of approximate equality, the results might still fail to meet the minimal standards of sociability that a reciprocal perspective would specify» (Gutmann, Thompson, 1996, p. 58).

54. Comme il s'agit d'une variante du choix rationnel, il va de soi que l'intérêt jouit d'une primauté théorique. 


\section{$340 \cdot$ Philosophiques / Automne 2002}

d'H abermas : I'agent s'engage à respecter les principes de vérité propositionnelle, de justesse normative et de sincérité55. Le mécanisme fondamental à l'œuvre ici est que dans tout débat public, les participants sont aux prises avec une norme sociale favorisant l'argumentation fondée sur la raison. Pour Elster, il s'agit plus que d'une simple norme, ce type de comportement se veut quasiment une « vérité conceptuelle» ${ }^{56}$. II existe une relation fondamentale, inaliénable entre le débat public et la raison: "To say, in a public debate, 'We should choose policy A because it is good for me', is to show a fundamental lack of understanding of what it means to offer an argument for something ${ }^{57}$. Toutefois, nous pouvons nous en tenir à la norme sociale sans que cela n'inquiète notre propos, car la frontière entre une norme sociale forte et une «quasi-vérité» est plutôt ténue; Elster l'admet également.

Résumons très brièvement la conception d'Elster des normes sociales. Celles-ci se fondent sur deux émotions, prenant ainsi deux formes distinctes: la honte, une auto-évaluation négative de sa personne et la culpabilité, une auto-évaluation négative d'un geste commis. La honte est provoquée par le mépris d'autrui suite à la violation d'une norme sociale forte, alors que la culpabilité émane du jugement négatif, provenant de soi ou d'autrui, d'un geste répréhensible, impliquant une norme sociale de moindre importance ${ }^{58}$. Ces émotions négatives nous portent à redéfinir nos actions de manière à éviter la douleur psychologique associée. Elles peuvent induire la transmutation d'une motivation (intérêt, raison ou passion) en une autre, ou encore sa fausse représentation (misrepresentation) ${ }^{59}$. La transmutation représente un conflit entre le désir de promouvoir son intérêt personnel et celui de maintenir une certaine estime de soi (positive self-image) ${ }^{60}$. C'est ici que l'on retrouve la possibilité de «sensibilité morale» de Rawls, si par «image positive» on considère le comportement raisonnable - au sens délibératif - dans I'agora $^{61}$. La fausse représentation se veut une variante hypocrite de la transmutation, les motivations n'y sont pas transformées au sens fort où la motivation originale cède sa place à une autre, elle est ici simplement masquée, maquillée afin de bien paraître au yeux d'autrui ${ }^{62}$. Dans bien des cas, nous

55. Elster, 1999, p. 337.

56. Elster, 1999, p. 372.

57. Elster, 1999, p. 373, italiques originales.

58. Elster, 1999, p. 145-56.

59. Elster, 1999, p. 332.

60. Elster, 1999, p. 369.

61. Pour que cette proposition soit vraiment solide, il faudrait démontrer empiriquement qu'un bon nombre de citoyens jouissent de ce type d'idéal personnel. $\mathrm{N}$ ous ne nous engagerons pas ici dans un tel travail, toutefois nous pouvons aisément constater qu'une telle disposition existe bel et bien, et d'une manière assez fréquente.

62. Quoiqu'un mécanisme secondaire, la «force civilisatrice de l'hypocrisie», peut faire dériver la fausse représentation vers une transmutation dans le cas des débats publics, en incorporant à la longue dans I'« image de soi » les déclarations faussement raisonnables de l'agent. Personnellement je crois que l'on surévalue grossièrement l'importance de ce mécanisme. 
serons à des lieues de la «sensibilité morale», mais comme nous le verrons, il existe certaines classes de situations où la fausse représentation peut se montrer compatible avec les propositions de la démocratie délibérative.

Ce qui nous intéresse pour notre propos, ce sont les transmutations et les fausses représentations de l'intérêt en raison. En soi, la transmutation présente des similitudes avec le modèle du citoyen que propose la démocratie délibérative, soit l'agent doté d'intérêts privés qui, au sein de la procédure délibérative, en vient à exprimer des propositions de nature impartiale et sensibles aux demandes d'autrui. A lors que la démocratie délibérative fonde le raffinement des préférences sur l'expression simple d'une certaine moralité politique a priori ainsi que sur les effets bénéfiques de la participation politique et du débat raisonnable, la stratégie de la transmutation cherche à localiser ce raffinement dans le profil psychologique de l'agent. Celle-ci aussi accepte l'idée de moralité a priori, mais précise de plus que la préférence exprimée par l'agent est le résultat d'un certain conflit interne entre cette moralité et ses intérêts privés. Les implications de ce niveau supplémentaire de formation de préférences sont attrayantes.

L'impartialité constitue la forme la plus commune de motivation orientée vers le collectif. Toutes les variantes de la démocratie délibérative l'exigent de ses citoyens idéaux. Pour Elster, l'impartialité se retrouve nécessairement dans toute conception sérieuse de la justice. Le problème, c'est qu'une infinité de variantes de justice respectent ce critère, et certaines correspondront plus à l'intérêt privé de l'agent que d'autres ${ }^{63}$. Cela ne signifie pas que l'agent choisit intentionnellement le concept de justice qui l'avantage le mieux (quoique la possibilité ne soit pas exclue), mais il est clair que la transmutation de l'intérêt en raison s'effectue plus aisément lorsque les deux exhibent des points communs. Avec le temps, une contrainte de cohérence s'impose : l'agent aura tendance à conserver les mêmes critères de justice dans différents contextes. $\mathrm{O} n$ ne peut à la fois croire sincèrement en un principe quelconque de justice et le modifier ou l'abandonner selon qu'il sert nos intérêts privés ou pas, cela irait à l'encontre de notre estime de soi64. Le sentiment de honte associé à une baisse de notre estime de soi nous force en quelque sorte à respecter nos propres critères de justice.

La fausse représentation des préférences est également reliée aux normes sociales. Elle émane d'une pression sociale à se comporter correctement dans l'agora. II est essentiel de noter que la norme conduit l'individu à paraître motivé par des considérations de justice, non à être sincèrement motivé. II peut masquer ses préférences pour deux raisons principales, le conformisme et la persuasion $^{65}$ (ce qui correspond aux deux mécanismes délibératifs de formation de

63. Elster, 1999, p. 339.

64. Elster, 1999, p. 343-49. II ne s'agit pas ici de la force civilisatrice de l'hypocrisie, car la croyance n'est pas hypocrite.

65. Si l'agent masque ses préférences par conformité, solidarité, etc., alors il internalise les principes délibératifs que les autres attendent de lui et cela devient une forme de transmutation - 
préférences impartiales, la censure de groupe et la persuasion). Cette première raison s'applique en vue d'éviter la désapprobation et le mépris d'autrui, causant respectivement de la culpabilité et de la honte, lorsque des propositions favorisant son propre intérêt sont présentées à une assemblée. Le simple fait de qualifier dès le départ une assemblée de «délibérative» - ou bien de marchandage, ou de simple vote - exerce une influence considérable sur le genre de propositions que l'on peut y émettre ${ }^{66}$. La persuasion ressemble beaucoup au conformisme; mais alors que ce dernier prend la forme négative d'une censure, la persuasion se sert de la raison comme avantage de négociation. Si l'agent croit qu'il sera plus en mesure d'obtenir ce qu'il veut de l'assemblée en maquillant ses propositions sous des traits raisonnables et impartiaux, alors il devient rationnel pour lui d'agir ainsi. Un cas intéressant concerne l'expression de menaces sous forme de mises en garde. Proférer une menace constitue une volonté d'usage de sa position de pouvoir, ce qui va à l'encontre de l'exigence de la «force du meilleur argument». En transformant intentionnellement une menace en mise en garde, celle-ci devient un argument raisonnable en bonne et due forme, pouvant être débattu ${ }^{67}$. Par exemple, au lieu de la menace du patron adressée à ses employés : «Si vous n'abandonnez pas vos revendications je vais effectuer des mises à pied», nous pourrions retrouver la mise en garde : «Si vous n'abandonnez pas vos revendications, le marché me forcera à effectuer des mises à pied ». La première proposition tire sa validité de la cré dibilité et de la position de pouvoir du locuteur, la seconde tire la sienne de la justesse de l'énoncé factuel.

En plus de la contrainte de cohérence, la fausse représentation fait face à une contrainte d'imperfection. La proposition raisonnable ne doit pas coïncider trop parfaitement avec les intérêts particuliers du locuteur, sinon on se doute de quelque chose. Idéalement l'agent devrait exprimer des positions raisonnables s'adressant à un large éventail de citoyens, pas seulement à lui et ses proches ${ }^{68}$. Un autochtone réclamant des ressources pour lui et son peuple sera moins respecté qu'un autre réclamant ces ressources pour tous les autochtones; cela même si dans les deux cas, le locuteur obtient la même part pour lui-même. Ces deux contraintes favorisent la sincérité de l'argument. Évidemment, plus l'argument apparaît sincère, en fait, plus il aura l'air d'une transmutation réelle plutôt que d'une représentation hypocrite, mieux il sera accepté par l'assemblée.

en fait il ne les masque plus, il les transforme. Bien sûr, si la solidarité demeure hypocrite, le cas de tromperie demeure aussi. N ous pouvons toutefois imaginer toutes sortes de situations où il n'est pas clair si nous sommes en présence de l'un ou de l'autre de ces deux mécanismes, ou d'une combinaison perverse des deux, étant donné que tout dépend du profil psychologique intime de l'agent. $M$ ais ce phénomène ne devrait pas nous empêcher d'élaborer des modèles de comportement valables, pas plus que les doutes soulevés quant au degré de rationalité des individus ne nous empêche de construire des modèles de choix rationnel valables. II s'agit d'être vigilants.

66. Elster, 1998, p. 100.

67. Elster, 1998, p. 100-4; Schelling, 1960

68. Elster, 1998, p. $104 ; 1999$, p. 376-77 
Il existe une autre forme de fausse représentation, nullement motivée par les émotions et les normes sociales. Ce sont les préférences stratégiques. Ici, l'agent trouve avantage à ne pas exprimer sa préférence réelle afin d'infléchir le résultat collectif en sa faveur, en utilisant ainsi les particularités de la procédure, en se «jouant» d'elle. Considérons l'exemple suivant. $0 \mathrm{n}$ demande à trois individus $(A, B, C)$ d'ordonner les options $x$, y et $z$ en précisant que dans l'agrégation, le premier choix vaudra 4 points, le second 3 points et le dernier 1 point. Les ordres de préférences sont les suivants: $A=[x, y, z], B=[x, y, z]$, $C=[z, y, x]$. Si l'individu C connait les préférences des deux autres et qu'il est persuadé qu'ils voteront honnêtement, il sera alors rationnel pour lui d'exprimer l'ordre $[y, z, x]$ car son vote « réel » donne comme résultat $R_{1}=(x=9$, $y=9, z=6)$ et le vote «stratégique» donne $R_{2}=(x=9, y=10, z=5)$. Comme $C$ préfère réellement $y$ à $x$ et que $z$ ne peut pas gagner, il devient rationnel de voter stratégiquement. Ce qu'il faut considérer, c'est que le choix de $C$ ne se fonde pas uniquement sur l'ordre primaire des alternatives, mais égal ement sur l'ordre secondaire des résultats possibles anticipés, soit $\left[R_{2}, R_{1}\right]$.

Le fardeau de la responsabilité représente une troisième forme de fausse représentation : je peux préférer une politique particulièrement pénible à appliquer sans vouloir passer pour celui par qui le malheur arrive. Dans un même ordre d'idée, les méthodes de votes à découvert (ou «main levée») peuvent inciter à l'hypocrisie; c'est pour cette raison que les votes secrets sont souvent privilégiés. Ce genre de comportement hypocrite survient en général lorsque le geste de voter ou de s'exprimer comporte des conséquences au-delà du débat social en cours. Pour revenir au vote à main levée, ce geste, en plus de signaler une prise de position, a une influence certaine sur les relations futures avec les concitoyens pouvant observer le geste, conséquences qui se répercuteront en d'autres circonstances. Par conséquent, l'électeur rationnel votant de cette façon doit considérer dans son calcul d'utilité non seulement le résultat du processus de décision, mais aussi les externalités découlant du simple geste, ce qui peut faire pencher la balance en faveur d'une fausse représentation de son choix.

La démocratie délibérative n'accepte pas la fausse représentation, en particulier le vote stratégique. Elle y voit une insincérité et un manquement général aux préceptes du citoyen raisonnable et accuse fréquemment la théorie du choix social de permettre ce genre de comportement. En revanche, plusieurs théoriciens du choix social se sentent mal à l'aise avec ce phénomène et cherchent à en minimiser la portée. II faut d'abord se demander en quoi le vote stratégique discrédite le choix social. La théorie se fonde sur une conception rationaliste de l'individu et le vote stratégique correspond parfaitement au type de comportement auquel on doit s'attendre d'un tel individu. Dans la plupart des cas, l'apparition de ce phénomène nous rappelle simplement que le geste de voter ne se déroule pas en vase clos. Pour conserver sa cohérence, la théorie devrait accepter toutes les conséquences découlant du choix rationnel, incluant les ordres secondaires de préférences et les externalités. La démo- 
cratie délibérative n'a pas non plus à rejeter la fausse représentation. Des trois types mentionnés, le premier peut inciter, de façon certes imparfaite, le comportement raisonnable par des normes sociales d'impartialité et de civisme; le second peut contribuer à contrer des alternatives inacceptables ${ }^{69}$ et le troisième est inévitable pour les théories qui s'intéressent au fonctionnement de la délibération publique et transparente.

$\mathrm{N}$ ous pourrions rapprocher le concept de normes sociales de celui de la «rationalité de rôle» 70 stipulant que nos critères d'évaluation rationnelle dépendent du contexte; dans le cas présent, il y aurait une distinction entre une rationalité «politique» exercée dans l'agora et une rationalité plus instrumentale exercée dans le marché. II s'agit d'une forme d'extension du choix rationnel orthodoxe, spécifiant que la satisfaction de préférences égoïstes n'est pas la seule avenue possible et que les agents sont suffisamment intelligents pour réfléchir en termes bénéfiques pour la société lorsqu'ils se retrouvent dans une situation décisionnelle pouvant affecter cette société. Plusieurs théoriciens se sont penchés sur cette rationalité politique. Pour Sunstein, les «préférences politiques» respectent les aspirations collectives et ont un penchant pour l'altruisme et la sympathie ${ }^{71}$. Elles peuvent également prendre la forme de «méta-préférences», des préférences pour des types de préférences (vouloir être impartial, par exemple). Boudon offre le concept de «rationalité axiologique» : sous certaines circonstances, la raison se fonde sur des critères nonconséquentialistes de moralité et de justice ${ }^{72}$. O $\mathrm{n}$ retrouve chez $\mathrm{H}$ arsanyi une théorie générale tripartite du comportement rationnel, se subdivisant en théorie de l'utilité, théorie des jeux et théorie éthique ${ }^{73}$. Cette dernière se veut une théorie des jugements de valeurs moraux rationnels. Les préférences y sont fondées sur l'impartial ité et l'agent cherche à maximiser l'utilité moyenne de la collectivité. Enfin, Sen cherche à compléter la théorie du choix rationnel par les concepts de «sympathie», I'inclusion de l'utilité d'autrui dans sa propre utilité, et d' 'engagement», soit les considérations morales dans la formation de l'utilité. Pour lui l'usage de la moralité demeure contextuelle, différentes problématiques collectives amèneront différentes positions morales ${ }^{74}$.

Un autre phénomène favorisant la discussion raisonnable consiste en ce qu'on pourrait nommer le «voile d'ignorance temporel » : même si les individus connaissent leur position sociale, leurs ressources, etc., une incertitude demeure toujours quant à l'avenir, ce qui les force jusqu'à un certain point à

69. Prenons exemple sur les élections présidentielles françaises de 2002. A u premier tour, un électeur de gauche qui n'avait pas l'intention de voter pour Lionel Jospin peut décider de «faussement » voter pour lui lorsqu'il apprend que J ean-M arie Le Pen a des chances de passer au second tour. Ce geste a toutes les allures d'un vote stratégique et pourtant, il est moralement irréprochable.

70. Goodin, 1986, p. 88-89.

71. Sunstein, 1993, p. 208-9.

72. Boudon, 1998, p. 191.

73. H arsanyi, 1990, p. 278.

74. Sen, 1982 , p. 91-93, p. 98-99. 
adopter des positions ne favorisant pas leur statut au détriment des autres. $\mathrm{Ce}$ point est encore plus vrai lorsque l'individu prend à cœur les intérêts de ses proches et de sa descendance. N ous obtenons une coïncidence entre l'intérêt particulier (possiblement altruiste) et l'impartialité75.

\section{2 - Les comportements stratégiques}

Le qualificatif «stratégique» désigne les actions individuelles, entreprises dans le cadre d'une décision collective, autres que le geste direct de voter ou de donner son opinion comme tel. Ces actions prennent une tournure indirecte, souvent considérée comme insidieuse. II n'en est pas toujours le cas. Avec le phénomène de la fausse représentation, nous avons vu que les comportements stratégiques ne sont pas nécessairement incompatibles avec la recherche collective de la bonne société. En plus du vote stratégique consistant en une réorganisation par l'individu de ses préférences sur la base des résultats agrégés anticipés, nous allons maintenant nous intéresser à deux autres formes de stratégie : le choix de la procédure et le marchandage de votes.

Le phénomène d'ambiguïté, bien connu dans la littérature du choix social, stipule qu'un même ensemble de choix individuels peut générer des résultats collectifs différents tout dépendant de la procédure adoptée, ce qui créé des opportunités de manipulation de la part de ceux contrôlant l'ordre du jour politique. M ême des citoyens parfaitement raisonnables se retrouveront aux prises avec cette difficulté car il n'existe pas de procédure parfaitement objective, mais dans leur cas on ne suppose pas que les intérêts personnels auront une influence sur le choix. N ous rencontrons dans les régimes démocratiques des procédures majoritaires dont le seuil de validité varie en fonction de l'importance de l'enjeu; par exemple, la majorité requise pour un amendement constitutionnel est plus élevée que pour l'adoption de lois ordinaires. On peut y voir une certaine sensibilité morale à vouloir conférer une importance spéciale aux institutions fondatrices libérales. Une autre explication, compatible avec la précédente, serait de protéger stratégiquement, selon le principe rationnel du « voile d'ignorance temporel », ces institutions contre l'exploitation par les générations suivantes ${ }^{76}$. On s'aperçoit que le choix stratégique n'est pas nécessairement immoral.

L'ambiguïté causée par la multiplicité des procédures agrégatives peut se voir en partie atténuée par une délibération portant sur la procédure particulière de vote permettant de trancher les débats si, bien sûr, les participants décident de passer au vote plutôt que de rechercher le consensus à tout prix. Ceci n'élimine pas le problème fondamental de l'ambiguïté, mais permet au moins une justification reconnue par tous de la procédure adoptée. La

75. Shepsle, 1989, p. 138-39; Elster, 1998, p. 114-16.

76. Elster, 2000, ch. II. 
justification demande toutefois que les individus placent le respect de l'idéal démocratique au-dessus de leurs intérêts personnels ${ }^{77}$.

Un cas intéressant concerne la distinction entre procédures agrégatives portant sur des prémisses et sur une conclusion ${ }^{78}$. Un exemple : un syndicat doit se décider sur le déclenchement d'une grève en spécifiant que si les membres jugent à la fois que les salaires sont trop bas et que les conditions de travail sont inadéquats (les prémisses), alors le mandat de grève sera valide (la conclusion). Supposons que $40 \%$ des membres croient que les salaires sont trop bas mais que les conditions sont adéquates, qu'un autre $40 \%$ croient exactement le contraire, et que les $20 \%$ restant croient les deux pré misses vraies. Pettit démontre aisément que si le vote porte sur les prémisses, soit deux votes distincts et une conclusion dérivée des résultats, le résultat favorisera la grève (car chaque prémisse remportera $60 \%$ ) tandis que si le vote porte sur la conclusion, la grève ne passera pas, remportant seulement $20 \%$. II est clair que le choix de la procédure est ici crucial. Laquelle est la plus juste? D'après Pettit, le vote sur prémisses semble plus juste car il respecte un critère fondamental (selon lui) de la démocratie délibérative, le débat sur des positions communes connues de tous ${ }^{79}$. M ais on peut le contrer en stipulant que seul $20 \%$ des membres désirent vraiment la grève. L'utilité d'un vote sur prémisse apparaît lorsque le groupe a besoin, en plus de répondre à une question affectant ses membres, de se constituer une « raison collective» ou, en d'autres termes, une ligne de parti. Le groupe peut ainsi offrir au public les raisons de sa décision. Les mérites de chacune des procédures dépendront du contexte et des buts visés par le groupe, et bien entendu la possibilité de manipulation stratégique demeure. Pettit ne réussit pas à nous convaincre que le vote sur prémisses est a priori préférable au vote sur conclusion, toutefois sa distinction demeure pertinente car on rencontre fréquemment ce genre de choix en politique.

L'échange de vote représente un autre champ stratégique en démocratie. II n'est pas nécessaire que les votes changent littéralement de mains, il s'agit simplement que les électeurs penchent d'un côté ou de l'autre selon certains incitatifs reçus. L'opinion populaire condamne ce genre de transaction; elle violerait le principe «une personne, un vote» et elle ouvrirait la porte aux abus de pouvoir, entre autre. Bien qu'en général, cette critique soit attrayante, il existe un argument soulignant le caractère potentiel lement équitable d'un tel échange. Dans sa conception «distributive» de la procédure, Christiano propose que la recherche de l'égal ité ne s'effectue pas au niveau de la procédure de vote, mais bien au niveau du processus global, soit le vote avec

77. M iller, 1992, p. 66.

78. Pettit, 2001.

79. Pettit, 2001, p. 2. Dans le même ordre d'idée, le vote sur conclusion incite à la «paresse» car il n'exige pas que l'individu fournisse les raisons de son choix (Pettit, 2001, p. 22). Cette critique porte sur les prémisses disjonctives, lorsqu'une seule prémisse vraie est suffisante pour que la conclusion soit vraie. 
tout ce qui l'entoure (collecte d'information, coalitions, etc.) ${ }^{80}$. Tout comme l'égal ité économique ne signifie pas que chacun possède exactement la même quantité de chaque bien mais plutôt une égalité globale sur l'ensemble des biens, l'égalité politique ne devrait pas porter sur l'égalité à chaque application de la procédure de vote mais plutôt sur l'égalité globale de l'ensemble des décisions collectives. II serait donc plus juste de permettre aux citoyens de marchander leur vote sur des sujets qui ne les intéressent pas. Toutefois, la justesse de ce principe dépend d'une distribution égalitaire des « ressources politiques», au dire même de l'auteur, afin d'éviter la possibilité d'abus de pouvoir. Sans la présence d'institutions prévenant ces abus et régulant le tout, cette forme d'égalité se rapproche de la «main invisible» en économie. Toutefois, l'échange de votes demeure un fait politique commun, même parmi des citoyens raisonnables, et ce phénomène n'est pas nécessairement injuste.

La délibération publique peut aussi être utilisée stratégiquement. Bien sûr, dans un tel cas, elle ne pourra être qualifiée de « raisonnable», I'usage stratégique de la délibération allant à l'encontre de tout ce que la démocratie délibérative propose. L'adoption de principes en public peut contribuer à une sympathie envers autrui et une volonté de remettre en question ses principes au nom de la recherche du consensus, c'est ce que la démocratie délibérative suggère, mais elle peut aussi provoquer exactement le contraire, soit un durcissement des positions. Le locuteur y met en jeu son honneur et sa réputation, $c^{\prime}$ est ce qui permet un tel durcissement ${ }^{81}$. Ce phénomène se veut particulièrement utile lors de situations présentant plusieurs forums, par exemple une délibération comprenant des moments à huis clos et en public. L'individu peut grandement améliorer sa position de négociation à huis clos en s'engageant publiquement au préalable à respecter certains principes. Dans une perspective de justice, l'engagement public peut constituer un instrument pré cieux de défense entre les mains de ceux occupant une position défavorable dans un débat inégal : «Q uand les puissants négocient en s'appuyant sur la force, les faibles le font en s'appuyant sur des principes ${ }^{82}$.

Cette section se voulait un bref survol des mécanismes pouvant engendrer la délibération en contexte rationnel. $\mathrm{N}$ ous avons voulu montrer que la formation et l'expression de préférences minimalement délibératives étaient possible chez des citoyens rationnels avec des mécanismes tels la transmutation, la fausse représentation, la rationalité de rôle ou encore le voile d'ignorance temporel. N ous avons remarqué aussi que les préférences stratégiques, un comportement rationnel inévitable, pouvaient avoir des conséquences positives pour la délibération raisonnable. Ces mécanismes sociaux nous ont permis d'entrevoir un vaste domaine sous-estimé autant par les théoriciens de la démocratie délibérative que du choix social. II y a encore beaucoup à dire

80. Christiano, 1993, p. 182-84.

81. Elster, 1994.

82. Elster, 1994, p. 244. 
sur la rationalité politique, les stratégies argumentatives, les relations de pouvoir, et bien plus encore. Tout de même, ce survol permet de nous rendre compte de l'importance capitale de ce champ d'étude de la démocratie que sont les interactions sociales en contexte mixte de rationalité, de normes sociales et de moralité.

\section{4 - Conclusion}

N ous avons constaté que la démocratie délibérative avait peine à appliquer les principes de justice sociale qu'elle propose. Élaborer des thèses de justice, aussi correctes soient-elles, n'est pas la même chose que d'en penser l'implé mentation, même dans des modèles abstraits. La démocratie délibérative a tendance à évacuer un peu trop rapidement les problèmes d'implémentation pour se concentrer sur les discussions de justice, ce qui constitue une lacune pour une théorie cherchant à créer une nouvelle façon de gouverner.

La discussion sur la délibération circonstancielle nous a permis d'établir la possibilité de délibération parmi des individus rationnels. Alors que de nombreux démocrates délibératifs relèguent les questions de formation et d'expression de préférences «raisonnables» à un mécanisme simpliste des vertus éducatrices et civilisatrices de la délibération, le modèle motivationnel de Elster va beaucoup plus loin et nous permet de découvrir un univers complexe de normes personnelles et sociales, et de comportements plus ou moins sincères. D'autres principes comme la rationalité de rôle et le « voile d'ignorance temporel » viennent préciser cette possibilité de délibération. De plus, certains comportements rationnels n'ayant que peu de familiarité a priori avec la délibération comme tel $n$ 'en demeurent pas moins compatibles avec les préceptes de la démocratie délibérative. $C$ 'est le cas notamment du vote stratégique, de la fausse représentation induite par le fardeau de la responsabilité et du choix de la procédure de vote lorsqu'un tel choix devient inévitable. La leçon à retenir ici est que le geste de délibérer (ou de voter) se déroule dans un contexte particulier qu'on ne peut ignorer. N ous avons vu avec l'exemple des présidentielles françaises que le vote stratégique est parfois nécessaire à la préservation d'une société juste. Le fardeau de la responsabilité nous invite à considérer les externalités liées à l'acte de délibération. La procédure «juste» de délibération ou d'agrégation varie d'une situation à l'autre.

Dans cet article, nous n'avons pas voulu placer les principes de la démocratie délibérative au banc des accusés. II ne s'agit nullement d'une critique au niveau des principes de justice, mais si la démocratie délibérative désire appliquer ses principes, alors elle doit prendre au sérieux les mécanismes sociaux rationnels que nous avons proposés. On ne peut admettre du même souffle des effets pervers de groupe (qui ne relèvent pas simplement de l'égoïsme) et souhaiter qu'ils disparaissent avec des citoyens raisonnables. Rawls semble l'avoir compris jusqu'à un certain point, c'est pour cette raison qu'il sépare son modèle idéal de «position originelle» de la discussion sur l'application de ce 
modèle, comprenant de nombreux postulats socio-politiques. Plusieurs démocrates délibératifs lui ont reproché de n'appliquer son modèle que dans les cas constitutionnels essentiels ${ }^{83}$. II faut peut-être y voir une certaine prudence théorique. En conclusion, la délibération circonstancielle fait apparaître la théorie du choix rationnel - en particulier la version proposée par Elster comme une théorie de l'individu à la fois utile pour l'implémentation des principes de justice et pas forcément anti-délibérative. La distinction entre l'individu rationnel et le citoyen raisonnable n'y apparaît plus aussi claire.

\section{Bibliographie}

Benhabib, Seyla, «Toward a D eliberative M odel of Democratic Legitimacy », dans Benhabib, S., dir., D emocracy and D ifference, Princeton, Princeton Univ. Press, 1996.

Boudon, Raymond, "Social M echanisms Without Black Boxes», dans H edström, P., Swedberg, R., dir., Social M echanisms, N ew York, Cambridge Univ. Press, 1998.

Christiano, Thomas, «Social Choice and Democracy», dans Copp, D., Hamilton, J., Roemer, J., dir., The I dea of D emocracy, N ew York, Cambridge Univ. Press, 1993.

Cohen, Joshua, «An Epistemic Conception of Democracy», Ethics, vol. 97, 1986, pp. 26-38.

, «D eliberation and D emocratic Legitimacy», dans Hamlin, A., dir., The Good Polity, Oxford, Blackwell, 1989.

, «Procedure and Substance in Deliberative Democracy», dans Benhabib,

S., dir., D emocracy and D ifference, Princeton, Princeton Univ. Press, 1996.

Cooke, M aeve, «Five A rguments for Deliberative Democracy», Political Studies, vol. 48, 2000, pp. 947-69.

Elster, J ohn, «The M arket and the Forum », dans Elster, J., H ylland, A., dir., Foundations of Social Choice Theory, N ew York, Cambridge Univ. Press, 1986.

, The Cement of Society, N ew York, Cambridge Univ. Press, 1989. " A rgumenter et négocier dans deux assemblées constitutives», R evue française de science politique, vol. 44, 1994, pp. 187-256.

" Deliberation and Constitution M aking», dans Elster, J., dir., D eliberative D emocracy, N ew York, Cambridge Univ. Press, 1998.

, Alchemies of the M ind, N ew York, Cambridge Univ. Press, 1999.

, U lysses U nbound, N ew York, Cambridge Univ. Press, 2000.

Goodin, Robert E., «L aundering Preferences», dans Elster, J., H ylland, A., dir., Foundations of Social Choice Theory, N ew York, Cambridge Univ. Press, 1986.

83. Cooke, 2000, p. 957-67; Benhabib, 1996, p. 75. 
Gutmann, Amy et Thompson, Dennis, «M oral Disagreement in a Democracy », Social Philosophy and Policy, vol. 12, 1995, pp. 87-110.

, D emocracy and D isagreement, Cambridge (M A), Belknap, 1996. , «Why Deliberative Democracy is Different», Social Philosophy and Policy, vol. 17, 2000, pp. 161-180.

Harsanyi, J ohn C., "Advances in Understanding Rational Behavior», dans M oser, P.K., dir., Rationality in Action, $\mathrm{N}$ ew York, Cambridge Univ. Press, 1990.

Johnson, James, «Arguing for Deliberation : Some Skeptical Considerations», dans Elster, J., dir., D eliberative D emocracy, N ew York, Cambridge Univ. Press, 1998.

Knight, Jack et Johnson, James, «Aggregation and Deliberation : O n the Possibility of Democratic Legitimacy », Political Theory, vol. 22, 1994, pp. 277-296.

Luban, D avid, «The Publicity Principle», dans Goodin, R., dir., The Theory of Institutional D esign, N ew York, Cambridge Univ. Press, 1996.

M iller, David, «D eliberative D emocracy and Social Choice», Political Studies, vol. 40, 1992, pp. 54-67.

Pettit, Philip, «D eliberative Democracy and the Dircursive Dilemma », Australian $\mathrm{N}$ ational University, Social and Political Theory Working Paper W 11, 2001.

Rawls, John, A Theory of Justice, Cambridge (M A), Belknap Press, 1971. , L ibéralisme politique, Paris, PUF, 1995.

Schelling, Thomas C., The Strategy of Conflict, Cambridge (M A), H arvard Univ. Press, 1960.

Sen, A martya, "R ational Fools», dans Sen, A., Choice, Welfare and M easurement, Cambridge (M A), M IT Press, 1982.

«Foundations of Social Choice Theory : An Epilogue», dans Elster, J., $\mathrm{H}$ ylland, A., dir., Foundations of Social Choice Theory, N ew York, Cambridge Univ. Press, 1986.

Shepsle, K enneth A., "Studying Institutions : Some Lessons From the R ational Choice A pproach », J ournal of Theoretical Politics, vol. 1, no. 2, 1989, pp. 131-47.

Sunstein, Cass R., «Democracy and Shifting Preferences», dans Copp, D., Hamilton, J., Roemer, J., dir., The Idea of D emocracy, N ew York, Cambridge Univ. Press, 1993.

Young, Iris $\mathrm{M}$ arion, «Communication and the $\mathrm{O}$ ther : Beyond Deliberative Democracy », dans Benhabib, S., dir., Democracy and Difference, Princeton, Princeton Univ. Press, 1996. 